\title{
Prediction of biological behavior and prognosis of colorectal cancer patients by tumor MSI/MMR in the Chinese population
}

This article was published in the following Dove Press journal:

OncoTargets and Therapy

8 December 2016

Number of times this article has been viewed

\author{
Wen-Yue Yan ${ }^{1, *}$ \\ jing $\mathrm{Hu}^{1,2, *}$ \\ $\mathrm{Li} \mathrm{Xie}^{2}$ \\ Lei Cheng ${ }^{2}$ \\ Mi Yang ${ }^{2}$ \\ $\mathrm{Li} \mathrm{Li}^{2}$ \\ Jiong $\mathrm{Shi}^{3}$ \\ Bao-Rui Liu ${ }^{2}$ \\ Xiao-Ping Qian ${ }^{1,2}$
}

'The Comprehensive Cancer Center, Nanjing Drum Tower Hospital Clinical College of Traditional Chinese and Western Medicine, Nanjing University of Chinese Medicine, ${ }^{2}$ The Comprehensive Cancer Center of Drum Tower Hospital, Medical School of Nanjing University and Clinical Cancer Institute of Nanjing University, ${ }^{3}$ Department of Pathology, Drum Tower Hospital, Medical School of Nanjing University, Jiangsu, People's Republic of China

*These authors contributed equally to this work

\begin{abstract}
Colorectal cancers (CRCs) exhibiting microsatellite instability (MSI) have special biological behavior. The clinical predictors for MSI and its survival relevance for the Chinese population were still unclear. Seven hundred ninety-five CRC patients were retrospectively assessed. Mismatch repair (MMR) proteins (MSH2, MSH6, PMS1, and MLH1) expression was detected by immunohistochemistry using tumor tissues of all patients. DNA MSI status was analyzed by polymerase chain reaction in 182 samples randomly selected from the 795 cases. Among all CRC tumor tissues, 97 cases (12.2\%) were with an MMR protein-deficient (MMR-D) phenotype, whereas 698 cases $(87.8 \%$ ) were with an MMR proteins intact (MMR-I) phenotype. A total of 21 (11.5\%) CRCs were identified as having high microsatellite instability, 156 (85.7\%) tumors were having microsatellite stability (MSS), and five (2.7\%) were having low microsatellite instability. Importantly, MMR status was demonstrated to be moderately consistent with MSI status $(\kappa=0.845,95 \%$ confidence interval $[\mathrm{CI}] 0.721,0.969)$. Unconditional logistic regression analysis revealed age, number of lymph node, tumor diameter, and tumor site as predictors for MSI with a substantial ability to discriminate different MSI status by area under curve of $80.62 \%$ using receiver operation curve. Compared with MMR-I, MMR-D was an independent prognostic factor for longer overall survival (hazard ratio $=0.340,95 \%$ CI $0.126,0.919 ; P=0.034$ ). MMR-D is an independent prognostic factor for better outcome. Our results may provide evidence for individualized diagnosis and treatment of $\mathrm{CRC}$, but this will require further validation in larger sample studies.
\end{abstract}

Keywords: microsatellite instability, mismatch repair, sporadic colorectal cancer, clinicopathological features, prognosis

\section{Introduction}

Colorectal cancer (CRC) is the third most commonly diagnosed cancer globally. ${ }^{1}$ In China, the incidence and mortality of CRC continues to rise, both of which rank the fifth place among malignant tumors, mainly due to the adoption of Western lifestyle and the aging of the population. ${ }^{2}$ According to molecular phenotypes, CRC are classified as CMS1, CMS2, CMS3, CMS4, and "others". CMS1 tumors are 14\% of CRCs and represent as hypermutated, microsatellite unstable, and with strong immune activation. CMS2 (canonical, 37\%) presents as epithelial, chromosomally unstable, with marked WNT and MYC signaling activation. CMS3 (metabolic, 13\%) presents as epithelial, with evident metabolic dysregulation. CMS4 (mesenchymal, 23\%) has prominent transforming growth factor $\beta$ activation, strong invasiveness, and angiogenesis. And "others" (with mixed features, $13 \%$ ) represent a transition phenotype or intratumoral heterogeneity. ${ }^{3}$ Precise 
molecular phenotype classification of CRC may contribute significantly to personalized prognosis and therapy.

Microsatellite instability (MSI), mainly due to mismatch repair (MMR) system defection, accounts for $\sim 15 \%$ of CRCs. About $80 \%$ of MSI CRCs are sporadic colorectal cancer, and the others are hereditary nonpolyposis colorectal cancer (HNPCC). ${ }^{4}$ The main function of the MMR system is to identify and repair the mismatches that occurred during DNA replication, therefore ensuring genomic conservation and stability. The most common MMR genes are MSH2, MSH6, PMS2, and MLH1. MSI is classified into high (MSI-H), low (MSI-L), and stable (MSS) types by analyzing the microsatellite status. ${ }^{5}$ According to the expression of the MMR proteins, tumors are classified into two types: MMR proteins deficient (MMR-D) and MMR proteins intact (MMR-I). ${ }^{5}$ Patients with MSI-H CRC have distinct clinicopathological characteristics, compared with patients with MSS/MSI-L phenotype. MSI-H CRCs are more common in females, tend to have proximal location, poorly differentiated mucinous phenotype, and increased rates for peritumoral and intratumoral lymphocytic infiltration. ${ }^{6}$

MSI analysis by polymerase chain reaction (PCR) is the gold standard technique for assessing microsatellite status. The American National Cancer Institute recommended five DNA markers for MSI test, ${ }^{7}$ including BAT25, BAT26, D2S123, D5S346, and D17S250. A tumor can be defined as MSI-H, when two or more of the five markers in the tumor DNA are tested positive. The tumor is defined as MSI-L if only one marker is tested positive, and MSS if all five markers are tested negative. In addition, the status of MMR defection can also be detected by examining the loss of MMR proteins expression via immunohistochemical (IHC) analysis.

Great difference in patterns of $M M R$ gene mutations was observed among patients with different ethnicity and geography. The $M S H 2$ gene has the highest mutant frequency in European and American populations, whereas MLH1 mutation was predominant in those from Japan and South Korea. ${ }^{8}$ MSH2 and MSH6 gene mutations occurred most frequently in Australians. ${ }^{9}$ The percentage of right colon cancer and mucinous adenocarcinoma in the Chinese population was lower than the Caucasian population, and there was great disparity in the microsatellite status between Chinese and Western populations. ${ }^{10,11}$ Few studies have focused on the relationship between MMR and MSI in China, and the clinical outcome relevance of MMR/MSI in Chinese population is still unclear. In order to find out the role of MSI and MMR system in Chinese CRC patients, this study first investigated the consistency in the technology matters (eg, sensitivity and specify) between PCR and IHC, and second, revealed the association of MMR defection with patients' outcome.

\section{Materials and methods}

\section{Patients}

We retrospectively identified 795 patients who had CRC surgery at the Affiliated Drum Tower Hospital of Nanjing University Medical School between January 2012 and July 2015, who then were enrolled as subjects in this study. This study was approved by the Medical Ethics Committee of the Affiliated Drum Tower Hospital of Nanjing University Medical School and all participants provided written informed consent. All patients satisfied the following criteria: they had 1) sporadic colon or rectal cancer confirmed by pathological diagnosis, 2) no preoperative therapy, including preoperative radiotherapy in the rectal cancer patients, 3 ) received radical resection or a palliative operation, and 4) clinical and pathological data available that can be extracted for analysis.

Exclusion criteria were 1) tumors in the appendix and anal canal; 2) genital tumor; 3) second primary tumor out of colorectal; 4) squamous cell carcinoma, melanoma, and gastrointestinal stromal tumor; and 5) in situ carcinoma (high-grade intraepithelial neoplasia).

Follow-up data were retrieved from medical records and confirmed by direct interviews with the patients' physicians or family members. Overall survival (OS) was defined as the interval between surgery and date of death. Progression-free survival (PFS) was the interval between surgery and recurrence or detection of metastasis. The postoperative period was measured from the surgery date to the time of the last follow-up or death. At the end of the study (January 2016), the median follow-up time for all patients was 15 months (range: 1-48 months).

\section{Tumor MMR proteins expression detected by IHC}

We conducted IHC analysis of MSH2, MSH6, PMS2, and MLH1 proteins with formalin-fixed paraffin-embedded tumor samples. After the tumor area adjacent to normal mucosa and/or lymphocytic infiltration was marked, $4 \mathrm{~mm}$ of paraffinized tissue was removed, and multiple tissue blocks were prepared. Finally, 4- $\mu \mathrm{m}$ thick sections were obtained for IHC following standard protocols. The mouse monoclonal antibodies used were anti-MSH2, anti-MSH6, anti-MLH1, and anti-PMS2 (BD Pharmingen). Adjacent normal tissues from each sample served as positive controls (Figure 1).

\section{Tumor MSI analysis detected by PCR}

For microsatellite analysis, DNA was extracted from formalin-fixed paraffin-embedded tissue with QIAamp Tissue kit (Qiagen) according to the manufacturers' instructions. 

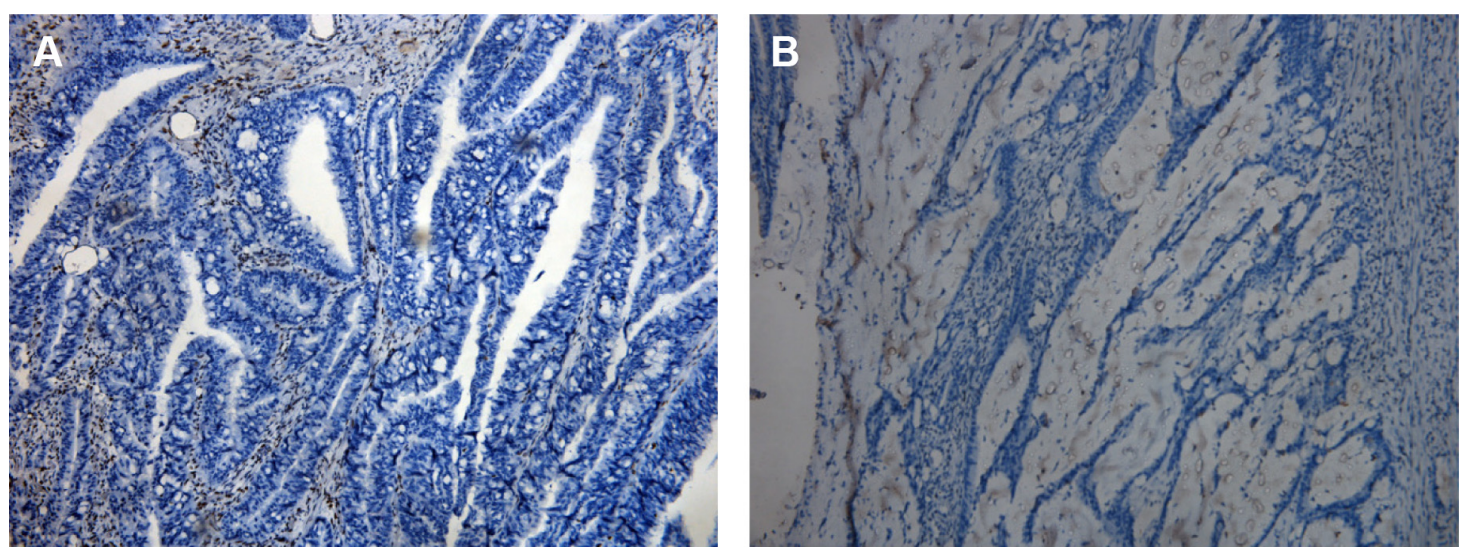

Figure I Immunohistochemical profile of mismatch repair gene protein. Notes: (A) MLHI negative $(\times 200)$. (B) PMS2 negative $(\times 200)$.

MSI status was evaluated with five microsatellite markers (BAT25, BAT26, D2S123, D5S346, and D17S250) using fluorescence-based PCR. DNA samples from tumor tissues and normal tissues were amplified in a $20 \mu \mathrm{L}$ volume containing $100 \mathrm{ng}$ of DNA, $1 \mu \mathrm{mol} / \mathrm{L}$ of dye-labeled forward and unlabeled reverse primers, $200 \mu \mathrm{mol} / \mathrm{L}$ of deoxynucleotide, $1.5 \mathrm{mmol} / \mathrm{L}$ of $\mathrm{MgCl}_{2}$, and $0.75 \mathrm{U}$ of Taq DNA polymerase. The PCR was performed under the following conditions: denaturation at $95^{\circ} \mathrm{C}$ for 5 minutes, 35 cycles of denaturation at $95^{\circ} \mathrm{C}$ for 30 seconds, annealing at $53^{\circ} \mathrm{C}$ for 30 seconds, and extension at $72^{\circ} \mathrm{C}$ for 30 seconds. Final extension was at $72^{\circ} \mathrm{C}$ for 10 minutes (Figure 2).

PCR product was analyzed by a genetic analyzer (Applied Biosystems 3500, ABI). Raw data were analyzed using GeneMapper 4.1 software. Patients were categorized as MSI-H if any two or more of the five studied markers showed positivity, MSI-L if only one marker showed positivity, and MSS if all markers showed negativity.
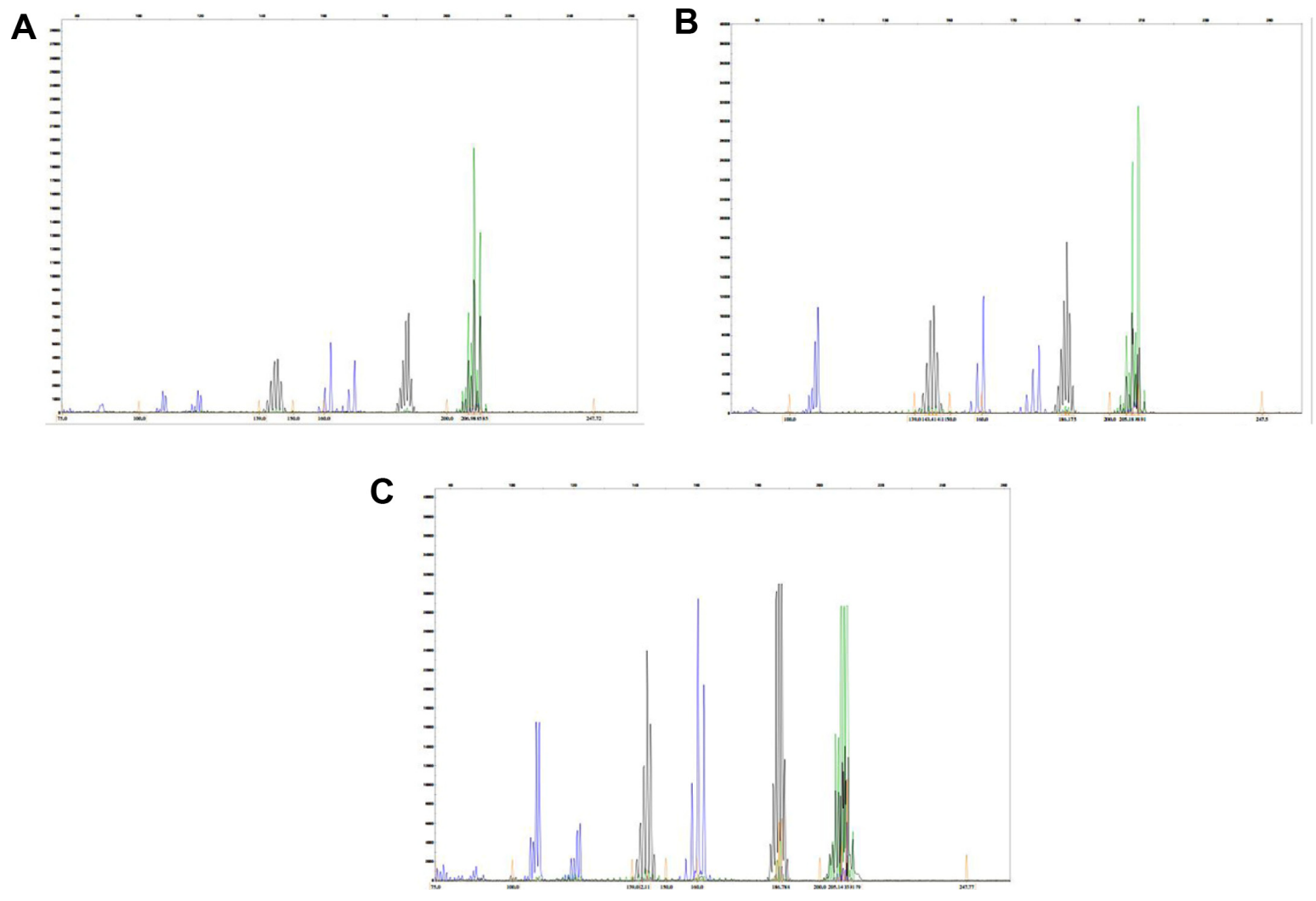

Figure 2 Images of separation of multiplex-amplified Bethesda loci (BAT25, BAT26, D2SI23, D5S346, and DI7S250).

Notes: (A) Normal amplification of all the five loci. (B) Representative image showing MSI-L. (C) Representative image showing MSI-H with arrows indicating instability. Abbreviations: MSI-H, microsatellite instability high; MSI-L, microsatellite instability low; MSS, microsatellite stable. 


\section{Statistical methods}

All statistical calculations were performed using SAS version 9.4 software (SAS Institute Inc., Cary, NC, USA). Unconditional logistic regression was used to identify potential predictors for MSI. Optimal cutoff value of each predictor was coded as $c$. The risk score was denoted as s and calculated as follows:

$$
S_{i j}=\left\{\begin{array}{l}
1, \mid v_{i j}>c_{j} \\
0, \mid v_{i j} \leq c_{j}
\end{array}\right.
$$

where $i$ is the $i$ th patient, and $j$ is the $j$ th clinical factor. To investigate the predictive value of clinical factors for MSI, we constructed a formula calculating the risk score function (RSF), according to the liner combination of the factors weighted by a univariate logistic regression coefficient. The RSF for the patient $i$ was calculated as follows:

$$
\mathrm{RSF}=\sum_{i}^{k} W_{i j} \times S_{i j}
$$

where $W_{i j}$ is the weight of the $j$ th clinical factor described previously. Then the RSF was analyzed as a continuous variable enrolling to the receiver operating curve (ROC). The area under the ROC curve (AUC) was used to estimate the predictive value of the clinical factors for MSI. PFS and OS were compared using the Kaplan-Meier method with a log-rank test. Risk of disease progression and death was calculated with Cox proportional hazards regression model. Differences were taken as significant when two-tailed $P$-value was $<0.05$.

\section{Results}

\section{Clinicopathological features of CRC}

A total of 795 CRC cases were included in this study; 482 of the patients $(60.6 \%)$ were males. Their age ranged from 15 to 91 years, and the median age of onset was 63 years. The location of primary tumors included proximal colon (30.4\%), distal colon (30.4\%), and the rectum (39.2\%). The clinicopathological data are summarized in Table 1.

\section{Frequency of the loss of MMR proteins expression and MSI status}

MMR proteins of all samples were detected by IHC. If all of the four MMR proteins were positively expressed, tumor was defined as MMR-I. Otherwise, it was defined as MMR-D. In all CRC tumor tissues, 97 cases (12.2\%) were of MMR-D phenotype, and 698 cases $(87.8 \%$ ) were of MMR-I
Table I Clinicopathological information of the studied patients

\begin{tabular}{ll}
\hline Clinical feature & $\mathbf{n}(\%)$ \\
\hline Gender & \\
Male & $482(60.6)$ \\
Female & $3 I 3(39.4)$ \\
Age (years) & \\
$\leq 60$ & $322(40.5)$ \\
$>60$ & $473(59.5)$ \\
Location & \\
Proximal & $242(30.4)$ \\
Distal & $242(30.4)$ \\
Rectum & $3 I I(39.2)$ \\
TNM stages & \\
I & $87(10.9)$ \\
II & $307(38.6)$ \\
III & $357(44.9)$ \\
IV & $44(5.6)$ \\
Tumor grade & \\
GI & $208(26.2)$ \\
G2 & $547(68.8)$ \\
G3 + G4 & $40(5.0)$ \\
Histology type & \\
Mucinous & II6 (I4.6) \\
Nonmucinous & $679(85.4)$ \\
\hline
\end{tabular}

phenotype. In the MMR-D group, one protein expression loss occurred in 56 cases $(57.7 \%)$, two proteins expression loss in 31 cases (32.0\%), and three proteins expression loss in 7 cases (7.2\%). Only three cases (3.1\%) were observed, whose tumor had no expression for all of the four MMR proteins. The frequency of expression loss was identified in each of the four MMR proteins: MLH1 (6.3\%), MSH2 (2.6\%), MSH6 (3.0\%), and PMS2 (7.3\%). MSI status of 182 samples that selected randomly from all patients was detected by PCR. Twenty-one tumors (11.6\%) were with MSI-H phenotype, 5 (2.7\%) were with MSI-L, and 156 (85.7\%) were with MSS. The frequency of each MSI marker positives is listed in Table 2 .

\section{Consistency between IHC and PCR methods to detect the status of MSI}

PCR is the gold standard technique for MSI detection ${ }^{12,13}$ (Table 3). We compared the MSI status and MMR protein expression to evaluate the accuracy of IHC results

Table 2 The frequency of each microsatellite instability marker positive in the Bethesda panel

\begin{tabular}{llllll}
\hline MSI status & \multicolumn{6}{l}{ Microsatellite instability markers } \\
\cline { 2 - 6 } & BAT25 & BAT26 & D2S I23 & D5S346 & DI7S250 \\
\hline MSI-H & 14 & 8 & 12 & 6 & 10 \\
MSI-L & 0 & 0 & 2 & 1 & 2 \\
Total & 14 & 8 & 14 & 7 & 12 \\
\hline
\end{tabular}

Abbreviations: $\mathrm{MSI}$, microsatellite instability; $\mathrm{MSI}-\mathrm{H}$, microsatellite instability high; MSI-L, microsatellite instability low. 
Table 3 Clinicopathological characteristics of the MMR-I and MMR-D groups

\begin{tabular}{|c|c|c|c|c|c|c|c|c|c|}
\hline & \multicolumn{2}{|c|}{ Proximal colon } & \multirow[t]{2}{*}{$P$-value } & \multicolumn{2}{|c|}{ Distal colon } & \multirow[t]{2}{*}{$P$-value } & \multicolumn{2}{|l|}{ Rectum } & \multirow[t]{2}{*}{$P$-value } \\
\hline & MMR-I & MMR-D & & MMR-I & MMR-D & & MMR-I & MMR-D & \\
\hline Gender & & & 0.545 & & & 0.912 & & & 0.900 \\
\hline Male & $12 \mid$ & 24 & & 136 & 12 & & 168 & 21 & \\
\hline Female & 78 & 19 & & 86 & 8 & & 109 & 13 & \\
\hline TNM stages & & & 0.002 & & & 0.874 & & & 0.002 \\
\hline$I+I I$ & 92 & 31 & & 104 & 9 & & 132 & 26 & \\
\hline III + IV & 107 & 12 & & 118 & 11 & & 145 & 8 & \\
\hline Tumor grades & & & 0.243 & & & 0.557 & & & 0.085 \\
\hline GI & 10 & 5 & & 11 & 0 & & 13 & 1 & \\
\hline G2 & $|3|$ & 25 & & 155 & 13 & & 194 & 29 & \\
\hline $\mathrm{G} 3+\mathrm{G} 4$ & 58 & 13 & & 56 & 7 & & 70 & 4 & \\
\hline Histological types & & & 0.699 & & & 0.712 & & & 0.562 \\
\hline Mucinous & $4 I$ & 10 & & 27 & 3 & & 30 & 5 & \\
\hline Nonmucinous & 158 & 33 & & 195 & 17 & & 247 & 29 & \\
\hline Lymph nodes metastasis & & & 0.002 & & & 1.000 & & & 0.002 \\
\hline Negative & 92 & 31 & & 104 & 9 & & 132 & 26 & \\
\hline Positive & 107 & 12 & & 118 & 11 & & 145 & 8 & \\
\hline
\end{tabular}

Abbreviations: MMR-D, mismatch repair proteins deficient; MMR-I, mismatch repair proteins intact.

in discriminating MSI. The sensitivity and specificity of the method of IHC assay were $82.6 \%(19 / 23)$ and $98.7 \%$ (157/159), respectively (Table 4). The $\kappa$ value of the two methods was 0.845 (95\% confidence interval [CI] $0.721,0.969$ ), indicating a moderate consistency between the two methods.

\section{Difference in clinicopathological characteristic between the MMR-I and MMR-D groups in proximal colon, distal colon, and rectal cancers}

We found that in proximal colon and rectal cancers, tumors with an MMR-I phenotype were more likely to be of later stage (53.8\% vs $27.9 \%$ and $52.3 \%$ vs $23.5 \%$, respectively) and have a lower percentage of lymph nodes metastasis ( $46.2 \%$ vs $72.1 \%$ and $47.7 \%$ vs $76.5 \%$, respectively). However, we could not detect the significant difference in the distribution of stage and lymph nodes metastasis between distal colon tumors with different MSI status (Table 3).

Table 4 Correlation between the PCR method and the IHC array for MSI status in tumors of I82 CRC patients

\begin{tabular}{lllll}
\hline & MMR-D (n) & MMR-I (n) & Total & $\begin{array}{l}\text { Kappa consistency } \\
\text { test }\end{array}$ \\
\hline MSI-H (n) & 19 & 2 & 21 & $0.845(0.721,0.969)$, \\
$\begin{array}{l}\text { MSS and } \\
\text { MSI-L (n) }\end{array}$ & & 157 & 161 & $P<0.001$ \\
Total & 23 & 159 & 182 & \\
\hline
\end{tabular}

Abbreviations: CRC, colorectal cancer; IHC, immunohistochemistry; MMR-D, mismatch repair proteins deficient; MMR-I, mismatch repair proteins intact; MSI, microsatellite instability; MSI-H, microsatellite instability high; MSI-L, microsatellite instability low; MSS, microsatellite stable; PCR, polymerase chain reaction.

\section{Predictors of MSI based on the clinical factors}

Unconditional logistic regression revealed that younger age (odds ratio $[\mathrm{OR}]=0.970, P=0.034$ ), fewer involved lymph nodes ( $\mathrm{OR}=0.244, P=0.003)$, lager tumor diameter $(\mathrm{OR}=1.434, P<0.001)$, and proximal colon lesions (OR $=0.361, P<0.001$ ) were predictors for MSI, and the increased risk of MSI in the presence of these predictors held when gender, tumor stage, and tumor difference were adjusted as covariates in the logistic regression model (Table 5).

We used RSF weighed by coefficient of logistic regression to construct an ROC curve (Figure 3). An $80.62 \%$ of AUC was observed, indicating that the combination of these predictors being highly efficient can identify potential CRC populations with MSI.

Table 5 Logistic regression models to find out predictors for MSI

\begin{tabular}{|c|c|c|}
\hline act & $R(95 \% \mathrm{Cl}), P-$ & $\begin{array}{l}\text { Adjusted OR (95\% Cl), } \\
\text { P-value (multivariate) }\end{array}$ \\
\hline & 0.910 & 0.97 \\
\hline ender $r^{2}$ & $0.600(0.273,1.319), 0.204$ & $0.617(0.256,1.486), 0.282$ \\
\hline $\begin{array}{l}\text { or } \\
\text { eter }\end{array}$ & 001 & 1.3 \\
\hline umor site ${ }^{b}$ & $0.361(0.207$ & $0.419(0.223,0.784), 0.007$ \\
\hline $\begin{array}{l}\text { of lymph } \\
\text { de }\end{array}$ & 0.24 & 0.2 \\
\hline ifference $^{c}$ & 0.746 & 0.798 \\
\hline & $0.64 \mathrm{I}(0.392,1.047), 0.076$ & $0.923(0.344,2.576), 0.874$ \\
\hline \multicolumn{3}{|c|}{$\begin{array}{l}\text { Notes: aFemale is the reference. 'lt was defined as I, } 2,3 \text { enrolling to logistic } \\
\text { regression model. The corresponding site is proximate colon site, distal colon } \\
\text { site, and rectum. It was defined as I-5 enrolling to logistic regression model. The } \\
\text { corresponding difference level is from poor differentiation to high differentiation. } \\
\text { Abbreviations: Cl, confidence interval; MSI, microsatellite instability; OR, odds } \\
\text { ratio. }\end{array}$} \\
\hline
\end{tabular}




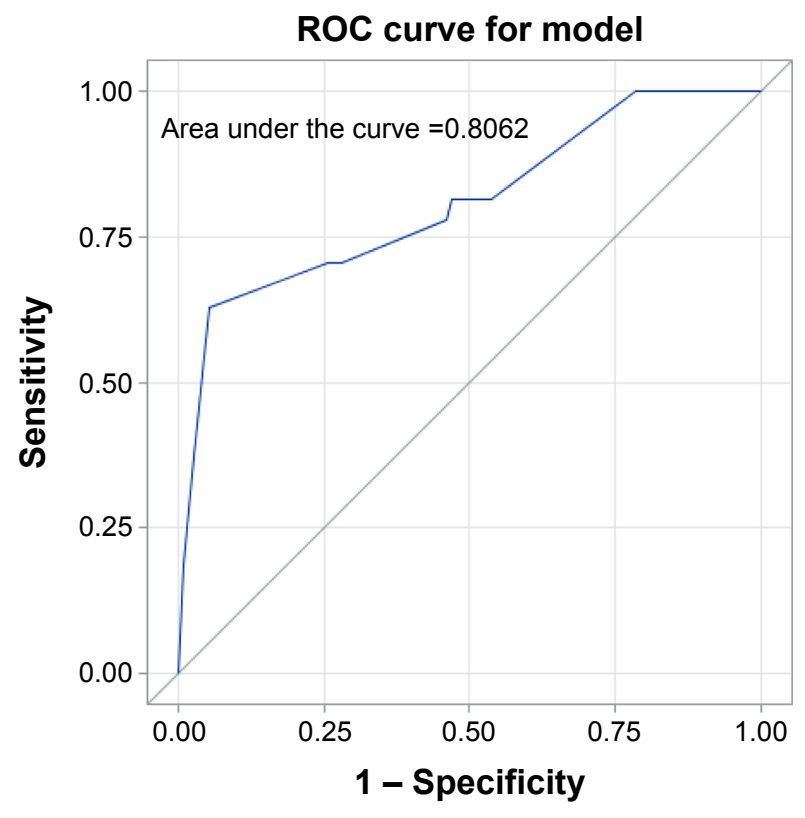

Figure 3 ROC curves for the predictive ability of clinical factors for microsatellite instability status.

Abbreviation: ROC, receiver operating characteristic curve.

\section{The role of MMR proteins expression and MSI status in prognosis of CRC patients}

Of all the patients, 711 patients were followed up by a median time of 15 months. A total of 166 patients (23.3\%), composed of 154 MMR-I and 12 MMR-D phenotype patients, had developed distant metastases and/or local recurrence. Sixtyeight patients (9.6\%) died, including 63 with the MMR-I and five with the MMR-D phenotype. Patients with MSI-H had longer PFS than those with MSS/MSI-L (log-rank test: $P=0.023$ ), although there was no significant difference in OS (Figure 4). In consistent with the MSI-based results, patients with MMR-D also had longer PFS and OS compared with those with MMR-I (log-rank test: PFS, 38.16 vs 29.12 months, $P=0.0011$ and $O S, 40.20$ vs 35.13 months, $P=0.013$; Figure 4). In the multivariate analysis, MMR-D was also found to be a significant indicator for OS, after adjustment for age, gender, TNM stage, tumor grade, tumor differentiation, tumor diameter, and tumor size (hazard ratio $[\mathrm{HR}]=0.340,95 \% \mathrm{CI} 0.126$, 0.919; $P=0.034$; Table 6).

Survival analysis was also conducted in the stage-based subgroups. Consist with the overall results, MSI-H had no impact on OS in stages II-III patients (log-rank test: $P=0.390$ ). However, the overall result that MSI-H was a predictor for prolonged PFS became insignificant when data were limited to stages II-III patients (log-rank test: $P=0.136$; Figure 5).
Because statistical power was largely decreased by subgroup analysis, we regarded this inconsistency with overall results as a bias caused by reduced sample size. However, it was revealed that stages II-III patients with MMR-D had longer OS and PFS, when compared with those with MMR-I (logrank test: OS, $P=0.011$; PFS, $P=0.025$; Figure 5). Importantly, MMR-D was also an independent predictor for longer PFS (HR $=0.402,95 \%$ CI $0.198,0.813 ; P=0.011)$ and OS ( HR $=0.231,95 \%$ CI $0.079,0.673 ; P=0.007)$, according to the multivariate COX regression analysis (Table 6).

\section{Discussion}

MSI is a change in DNA sequence of any length due to either insertion or deletion of repeating units in the genome. ${ }^{7}$ Because of the application of MSI for screening HNPCC, it has been paid more and more focus on studies in CRC. It may be used as a marker to select stage II CRC patients who will benefit from 5-fluorouracil adjuvant chemotherapy. ${ }^{14}$ Recent studies indicated that MSI status might be a particular candidate biomarker for checkpoint immunotherapy in CRC because patients with an MMR-D phenotype could benefit from immune checkpoint blockade with pembrolizumab. ${ }^{15,16}$ Three methods - IHC, PCR, and DNA sequencing - can be used to identify the MSI status of CRC. The IHC method can be used to detect the loss of expression of MMR proteins in the tumor tissue with lower cost and less difficulty. However, only a limited number of MMR proteins can be detected, and the results greatly depend on the individual pathologist. PCR is considered as the "gold standard" for the identification of MSI phenotype. ${ }^{12,13}$ It also has the advantage of having high reproducibility. DNA sequencing can be used to assess MSI status by detecting the mutations in the genes of enzymatic DNA repair, and it is the only way to identify novel mutations. ${ }^{17}$ However, DNA sequencing is time consuming, expensive, and requires specialized equipment. Therefore, a more accuracy and cost-effective test protocol is expected to arise from a combination of these methods. In our study, in consistent with previous studies, ${ }^{18-20}$ there was a moderate consistency between the IHC method for MMR detection and the PCR method for MSI assessment, indicating that combining the results of the IHC and PCR methods is a feasible strategy to obtain more promising results than that of considering either method alone. However, some tumors were MSI-H, but were classified as MMR-D, and some MSS were classified as MMR-D by IHC. Therefore, to get a more accurate determination of MSI status by IHC instead of PCR, additional markers 
A

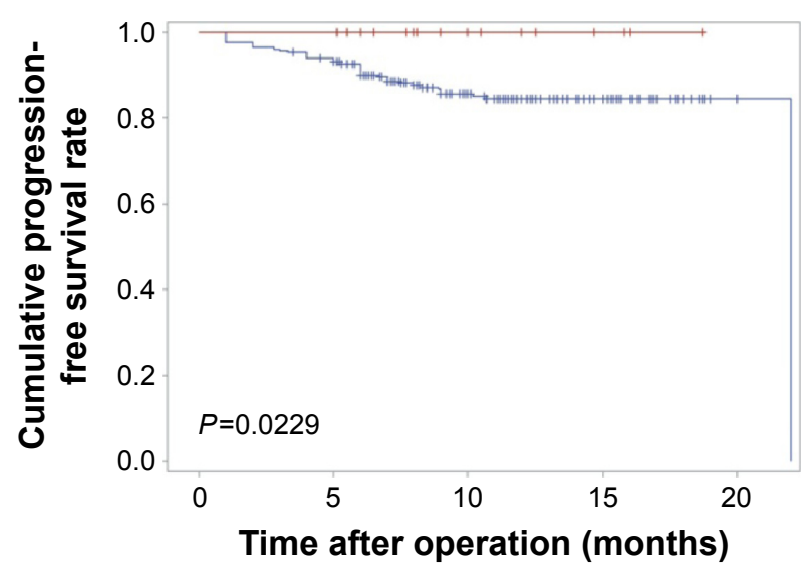

B

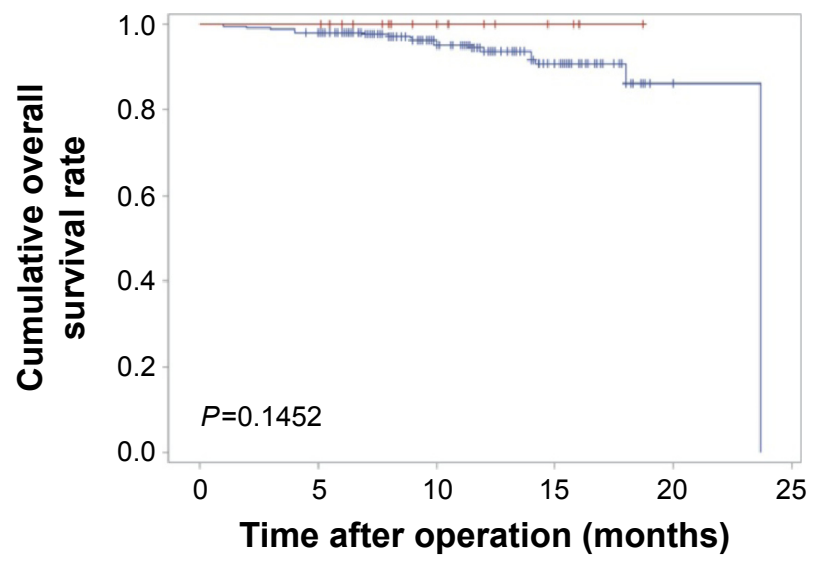

$-\mathrm{MSI}-\mathrm{H} \quad \mathrm{MSS}+\mathrm{MSI}-\mathrm{L}$

C

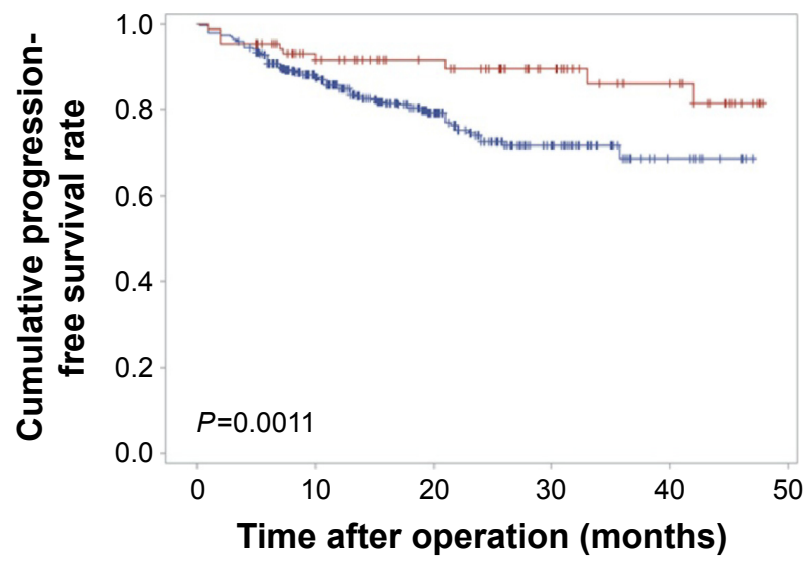

D

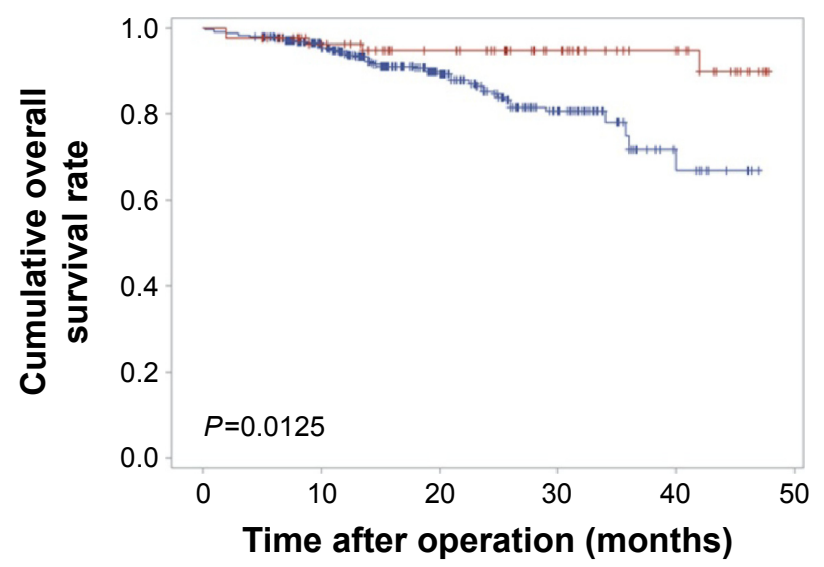

Figure 4 PFS and OS in all patients.

Notes: (A and B) PFS and OS according to MSI status in all patients. The red line is MSI-H. The blue line is MSS/MSI-L. (C and D) PFS and OS according to MMR proteins expression in overall patients. The red line is MMR-D. The blue line is MMR-I.

Abbreviations: MMR, mismatch repair gene; MMR-D, mismatch repair proteins deficient; MMR-I, mismatch repair proteins intact; MSI, microsatellite instability; MSI-H, microsatellite instability high; MSI-L, microsatellite instability low; MSS, microsatellite stable; PFS, progression-free survival; OS, overall survival.

Table 6 Cox's proportional hazards regression model for the correlation of MMR status with PFS and OS

\begin{tabular}{|c|c|c|c|c|c|c|c|c|}
\hline \multirow[t]{2}{*}{ Patients } & \multicolumn{4}{|l|}{ PFS } & \multicolumn{4}{|l|}{ OS } \\
\hline & HR (95\% Cl) & $P$-value & $\begin{array}{l}\text { Adjusted HR } \\
(95 \% \mathrm{Cl})\end{array}$ & $P$-value & HR (95\% Cl) & $P$-value & $\begin{array}{l}\text { Adjusted } \mathrm{HR}^{\mathrm{a}} \\
(95 \% \mathrm{Cl})\end{array}$ & $P$-value \\
\hline Overall & & 0.015 & & 0.088 & & 0.012 & & 0.034 \\
\hline MMR-D & $0.442(0.229-0.852)$ & & $0.547(0.274-1.093)$ & & $0.300(0.118-0.766)$ & & $0.340(0.126-0.919)$ & \\
\hline MMR-I & References & & & & & & & \\
\hline Stages II-III & & 0.030 & & 0.011 & & 0.017 & & 0.007 \\
\hline MMR-D & $0.464(0.232-0.926)$ & & $0.402(0.198-0.813)$ & & $0.282(0.100-0.797)$ & & $0.231(0.079-0.673)$ & \\
\hline MMR-I & - & & & & & & & \\
\hline
\end{tabular}

Note: aAjusted for age, gender, TNM stage, tumor grade, and tumor diameter.

Abbreviations: $\mathrm{Cl}$, confidence interval; HR, hazard ratio; MMR, mismatch repair gene; MMR-D, mismatch repair proteins deficient; MMR-I, mismatch repair proteins intact; OS, overall survival; PFS, progression-free survival. 
A

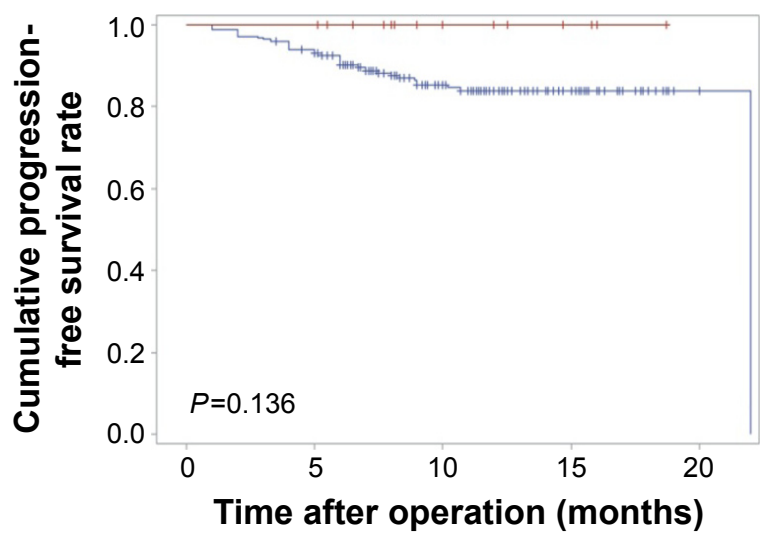

B

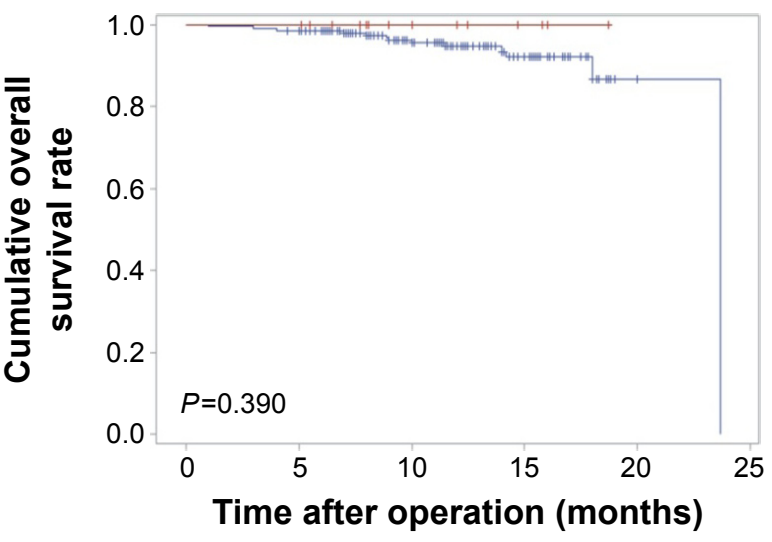

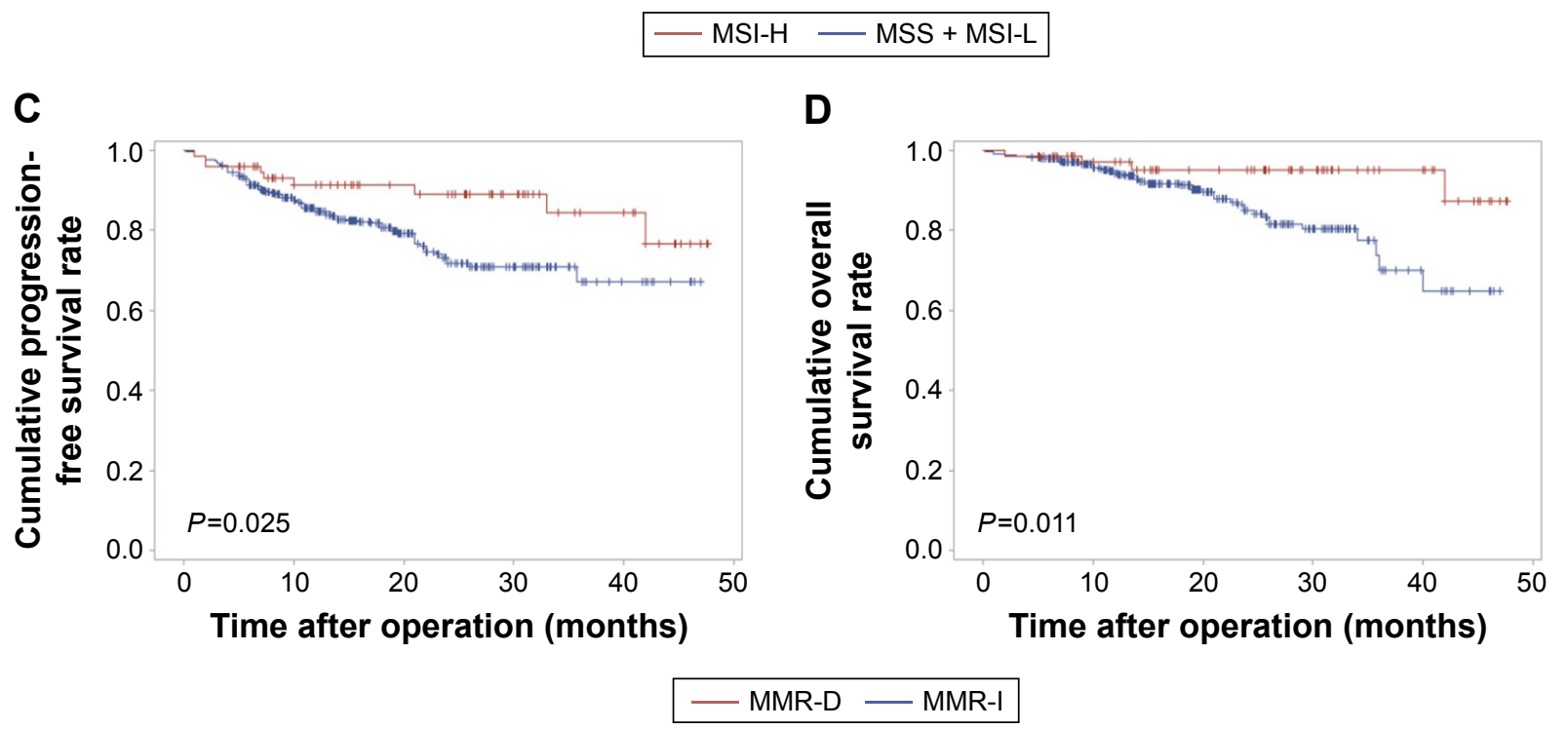

Figure 5 PFS and OS in patients with stages II-III.

Notes: (A and B) PFS and OS according to MSI status in patients with stages II-III. The red line is MSI-H. The blue line is MSS/MSI-L. (C and D) PFS and OS according to mismatch repair gene proteins expression in patients with stages II-III. The red line is MMR-D. The blue line is MMR-I.

Abbreviations: MMR-D, mismatch repair proteins deficient; MMR-I, mismatch repair proteins intact; MSI, microsatellite instability; MSI-H, microsatellite instability high; MSI-L, microsatellite instability low; MSS, microsatellite stable; PFS, progression-free survival; OS, overall survival.

should be tested, such as some other microsatellite loci (D18S65, D18S69, D17S1176, D17S796, D8S261, D8S254, and D8S550). ${ }^{21}$ Moreover, DNA sequencing may also be helpful in combination with PCR and IHC to produce more reliable results.

Studies showed that MSI tumors were more commonly located in the proximal colon. ${ }^{6,22}$ In this study, we also found that MMR-D and MSI-H type tumors predominantly occurred in the proximal colon. Moreover, we compared the clinicopathological factors of MMR-D and MMR-I type tumors in proximal colon, distal colon, and rectal cancers. In the proximal colon and rectal cancers, patients with the MMR-I type were found to be tilted to have later stage and have more lymph node metastasis. These different features may arise from a different carcinogenic pathway. There are some studies which reported that tumors with different MMR status may differ in the distribution of age, $\mathrm{T}$ stage, $\mathrm{N}$ stage, and different gender. ${ }^{22-24}$

We revealed that age, lymph node, tumor diameter, and tumor location were predictors for increased risk of MSI, and the increased risk held when gender, tumor stage, and tumor difference were adjusted. This result might help us to identify patients whose DNA MSI status are needed to be tested. Previous studies on the prognostic value of MSI for CRC patients have had controversial results. ${ }^{19,20,25-30}$ Patients inclucded in these studies were from Western-based populations. Although there were a few studies focusing on MSI/ MMR in Chinese population, ${ }^{25,31-33}$ the prognostic relevance 
of MMR and MSI are still unclear. In our study, with a substantial amount of sample size, we found that MMR proteins expression was an independent factor for prolonged PFS and OS. Studies showed that MSI and MMR had unequal prediction ability for prognosis in patients with different stages. It was indicated that MSI-H tumors was an indicator of good prognosis in stages II and III CRC patients, but survival cannot be significantly prolonged in the context of MSI-H in stage IV patients. ${ }^{21,34-37}$ Consistent with the previous findings, we also found that MMR-D was a marker for good prognosis in stages II and III. However, due to limited sample size, the prognostic value of MMR-D in stage IV patients could not be statistically calculated.

There are some limitations in this study. Only 68 patients achieved the end of survival events, indicating the limitation of a short follow-up time. These patients will continue to be followed up, and the survival data will be reanalyzed in the next study. Another limitation is the lack of the therapeutic information in this study, making us unable to clarify the interactive effect of therapy and MSI/MMR status on clinical outcome.

\section{Conclusion}

MMR protein expression detected by IHC has a moderate consistency with PCR in discriminating MSI status in CRC tumors. Age, lymph node, tumor diameter, and tumor location are strong predictors for DNA MSI status. MMR-D is an independent favorable prognostic factor for primary CRC patients. Our results may provide evidence for individualized diagnosis and treatment according to MSI status-based prediction, if validated by larger studies.

\section{Acknowledgments}

This study was supported by a grant from Nanjing City Committee of Science and Technology (201503013), a grant from the Natural Science Foundation of Jiangsu province (BK20161107), and a grant from Key project of Nanjing Municipal Health Bureau (zkx13022).

\section{Disclosure}

The authors report no conflicts of interest in this work.

\section{References}

1. Torre LA, Bray F, Siegel RL, Ferlay J, Lortet-Tieulent J, Jemal A. Global cancer statistics, 2012. CA Cancer J Clin. 2015;65(2):87-108.

2. Chen W, Zheng R, Baade PD, et al. Cancer statistics in China, 2015. CA Cancer J Clin. 2016;66(2):115-132.

3. Guinney J, Dienstmann R, Wang X, et al. The consensus molecular subtypes of colorectal cancer. Nat Med. 2015;21(11):1350-1356.
4. Boland CR, Goel A. Microsatellite instability in colorectal cancer. Gastroenterology. 2010;138(6):2073-2087.e2073.

5. Boland GM, Chang GJ, Haynes AB, et al. Association between adherence to National Comprehensive Cancer Network treatment guidelines and improved survival in patients with colon cancer. Cancer. 2013;119(8): $1593-1601$.

6. Ward R, Meagher A, Tomlinson I, et al. Microsatellite instability and the clinicopathological features of sporadic colorectal cancer. Gut. 2001;48(6):821-829.

7. Boland CR, Thibodeau SN, Hamilton SR, et al. A National Cancer Institute Workshop on Microsatellite Instability for cancer detection and familial predisposition: development of international criteria for the determination of microsatellite instability in colorectal cancer. Cancer Res. 1998;58(22):5248-5257.

8. Yearsley M, Hampel H, Lehman A, Nakagawa H, de la Chapelle A, Frankel WL. Histologic features distinguish microsatellite-high from microsatellite-low and microsatellite-stable colorectal carcinomas, but do not differentiate germline mutations from methylation of the MLH1 promoter. Hum Pathol. 2006;37(7):831-838.

9. Hall G, Clarkson A, Shi A, et al. Immunohistochemistry for PMS2 and MSH6 alone can replace a four antibody panel for mismatch repair deficiency screening in colorectal adenocarcinoma. Pathology. 2010; 42(5):409-413.

10. Lin J, Qiu M, Xu R, Dobs AS. Comparison of survival and clinicopathologic features in colorectal cancer among African American, Caucasian, and Chinese patients treated in the United States: results from the Surveillance Epidemiology and End Results (SEER) database. Oncotarget. 2015;6(32):33935-33943.

11. Cheah PL, Looi LM, Teoh KH, Rahman NA, Wong LX, Tan SY. Colorectal carcinoma in Malaysians: DNA mismatch repair pattern in a multiethnic population. Asian Pac J Cancer Prev. 2014;15(7):3287-3291.

12. Lech G, Slotwinski R, Krasnodebski IW. The role of tumor markers and biomarkers in colorectal cancer. Neoplasma. 2014;61(1):1-8.

13. Patil DT, Bronner MP, Portier BP, Fraser CR, Plesec TP, Liu X. A five-marker panel in a multiplex PCR accurately detects microsatellite instability-high colorectal tumors without control DNA. Diagn Mol Pathol. 2012;21(3):127-133.

14. Bendardaf R, Lamlum H, Ristamaki R, Korkeila E, Syrjanen K, Pyrhonen S. Thymidylate synthase and microsatellite instability in colorectal cancer: implications for disease free survival, treatment response and survival with metastases. Acta Oncol. 2008;47(6):1046-1053.

15. Xiao Y, Freeman GJ. The microsatellite instable subset of colorectal cancer is a particularly good candidate for checkpoint blockade immunotherapy. Cancer Discov. 2015;5(1):16-18.

16. Le DT, Uram JN, Wang H, et al. PD-1 blockade in tumors with mismatch-repair deficiency. $N$ Engl J Med. 2015;372(26):2509-2520.

17. Serrano M, Lage P, Belga S, et al. Bethesda criteria for microsatellite instability testing: impact on the detection of new cases of Lynch syndrome. Fam Cancer. 2012;11(4):571-578.

18. Amira AT, Mouna T, Ahlem B, et al. Immunohistochemical expression pattern of MMR protein can specifically identify patients with colorectal cancer microsatellite instability. Tumour Biol. 2014;35(7): 6283-6291.

19. Gupta S, Ashfaq R, Kapur P, et al. Microsatellite instability among individuals of Hispanic origin with colorectal cancer. Cancer. 2010;116(21): 4965-4972.

20. Yoon YS, Yu CS, Kim TW, et al. Mismatch repair status in sporadic colorectal cancer: immunohistochemistry and microsatellite instability analyses. J Gastroenterol Hepatol. 2011;26(12):1733-1739.

21. Lanza G, Gafa R, Santini A, Maestri I, Guerzoni L, Cavazzini L. Immunohistochemical test for MLH1 and MSH2 expression predicts clinical outcome in stage II and III colorectal cancer patients. $J$ Clin Oncol. 2006;24(15):2359-2367.

22. Thomas ML, Hewett PJ, Ruszkiewicz AR, Moore JW. Clinicopathological predictors of benefit from adjuvant chemotherapy for stage $\mathrm{C}$ colorectal cancer: microsatellite unstable cases benefit. Asia Pac J Clin Oncol. 2015;11(4):343-351. 
23. Hong SP, Min BS, Kim TI, et al. The differential impact of microsatellite instability as a marker of prognosis and tumour response between colon cancer and rectal cancer. Eur J Cancer. 2012;48(8):1235-1243.

24. Waldmann E, Ferlitsch M, Binder N, et al. Tumor and patient characteristics of individuals with mismatch repair deficient colorectal cancer. Digestion. 2015;91(4):286-293.

25. Meng WJ, Sun XF, Tian C, et al. Microsatellite instability did not predict individual survival in sporadic stage II and III rectal cancer patients. Oncology. 2007;72(1-2):82-88.

26. Kim HR, Kim HC, Yun HR, et al. An alternative pathway in colorectal carcinogenesis based on the mismatch repair system and p53 expression in Korean patients with sporadic colorectal cancer. Ann Surg Oncol. 2013;20(12):4031-4040.

27. Kang J, Lee HW, Kim IK, Kim NK, Sohn SK, Lee KY. Clinical implications of microsatellite instability in T1 colorectal cancer. Yonsei Med J. 2015;56(1):175-181.

28. Yoon YS, Kim J, Hong SM, et al. Clinical implications of mucinous components correlated with microsatellite instability in patients with colorectal cancer. Colorectal Dis. 2015;17(8):O161-O167.

29. Shin US, Cho SS, Moon SM, et al. Is microsatellite instability really a good prognostic factor of colorectal cancer? Ann Coloproctol. 2014; 30(1):28-34.

30. Tougeron D, Sickersen G, Mouillet G, et al. Predictors of disease-free survival in colorectal cancer with microsatellite instability: An AGEO multicentre study. Eur J Cancer. 2015;51(8):925-934.
31. Huang YQ, Yuan Y, Ge WT, Hu HG, Zhang SZ, Zheng S. Comparative features of colorectal and gastric cancers with microsatellite instability in Chinese patients. J Zhejiang Univ Sci B. 2010;11(9):647-653.

32. Li W, Zhi W, Zou S, et al. Distinct clinicopathological patterns of mismatch repair status in colorectal cancer stratified by KRAS mutations. PLoS One. 2015;10(6):e0128202.

33. Ye JX, Liu Y, Qin Y, Zhong HH, Yi WN, Shi XY. KRAS and BRAF gene mutations and DNA mismatch repair status in Chinese colorectal carcinoma patients. World J Gastroenterol. 2015;21(5):1595-1605.

34. Wright CM, Dent OF, Barker M, et al. Prognostic significance of extensive microsatellite instability in sporadic clinicopathological stage C colorectal cancer. Br J Surg. 2000;87(9):1197-1202.

35. Ribic CM, Sargent DJ, Moore MJ, et al. Tumor microsatelliteinstability status as a predictor of benefit from fluorouracil-based adjuvant chemotherapy for colon cancer. $N$ Engl J Med. 2003;349(3): 247-257.

36. Sargent DJ, Marsoni S, Monges G, et al. Defective mismatch repair as a predictive marker for lack of efficacy of fluorouracilbased adjuvant therapy in colon cancer. J Clin Oncol. 2010;28(20): 3219-3226.

37. Goldstein J, Tran B, Ensor J, et al. Multicenter retrospective analysis of metastatic colorectal cancer (CRC) with high-level microsatellite instability (MSI-H). Ann Oncol. 2014;25(5):1032-1038.
OncoTargets and Therapy

\section{Publish your work in this journal}

OncoTargets and Therapy is an international, peer-reviewed, open access journal focusing on the pathological basis of all cancers, potential targets for therapy and treatment protocols employed to improve the management of cancer patients. The journal also focuses on the impact of management programs and new therapeutic agents and protocols on

\section{Dovepress}

patient perspectives such as quality of life, adherence and satisfaction The manuscript management system is completely online and includes a very quick and fair peer-review system, which is all easy to use. Visit http://www.dovepress.com/testimonials.php to read real quotes from published authors. 